Arqueología y Sociedad,

№ 21, 2010

\title{
"Plateros" precoloniales tardíos en Tambo de Mora, valle DE ChINCHA (siglos XIV - XVI)*
}

\author{
Javier Alcalde Gonzáles, Carlos Del Águila Chávez, \\ Fernando Fujita Alarcón, Enrique Retamozo Rondón \\ Instituto Andino De Estudios Arqueológicos - INDEA**
}

\section{Resumen}

Durante los años 1988 y 2001, el Programa de Investigaciones Arqueológicas Chincha del Instituto Andino de Estudios Arqueológicos - INDEA, ha venido enfocando parte de sus tareas en un sector del conjunto monumental de Tambo de Mora compuesto por una superposición de restos de talleres metalúrgicos de purificación / calentamiento y trabajo de productos finales. Los talleres parecen corresponder a, por lo menos, tres momento grandes. En el interior de cada uno y en sus lapsos de uso se han efectuado remodelaciones y redistribuciones. Los estudios apuntan al trabajo de la plata y, probablemente, oro.

Palabras clave

Hornos, platería, metalurgia, moldes, Chincha, Costa Central Andina, Tambo de Mora, microscopía electrónica.

\begin{abstract}
During the years 1998 and 2001, The Chincha Archaeological Investigations Program, of the Andean Institute of Archaeological Studies - INDEA, has focused part of their investigations in a sector of the monumental complex of Tambo de Mora, composed by a superposition of evidences from metallurgical workshops. These were used to purify and heat the metal, and to making the final products. The use, there were remodelations and redistributions of this space. The analysis indicates that the metallurgical work was concentrated on silver and, probably, gold.
\end{abstract}

\section{Keywords}

Furnaces, silverware, metallurgy, moulds, Chincha, Andean Central Coast, Tambo de Mora, electron microscopy.

\footnotetext{
* Este trabajo fue publicado en Anales del Museo de América, 10, pp. 43-57, 2002, Madrid. Cabe indicar que la jefatura de los trabajos de campo de las temporadas 1999-2000, en el subsector "Hornos" estuvo a cargo de la arqueóloga Alejandra Figueroa Flores.

** Instituto Andino de Estudios Arqueológicos - INDEA, Programa de Investigaciones Arqueológicas Chincha, Proyecto Tambo de Mora. Con el apoyo del American Museum of Natural History.
} 
"Los Indios Yngas, mandaron los visitadores que pagasen de tribute ropa, algodón, coca, agí, pescado y otras cosas de frutas secas, todo en poca cantidad porque no tenían minas de oro y plata en su tierra, aunque fueron mui amigos de ella, y la poseían con sus grandes rescates que tenían con los Indios serranos, como se ha visto por la riqueza que se han hallado en Truxillo, Chincha, Ita y en otras partes de este Reyno."

"Os recomiendo el valle de Chincha con todos sus yndios e caciques e prencepales que le pertenecen y sus sujetos, con todos los mytimaes e plateros que tienen fuera de su tierra, sus sujetos y naturales del dicho valle..." (Archivo General de Indias, Justicia 1075).

\section{Antecedentes}

Son escasos los datos arqueológicos sobre actividad minera extractiva precolonial en Sudamérica, sobre todo si se comparan con los datos arqueológicos de metalurgia (fundiciones, objetos de trabajo, desechos, y productos), y con los datos etno-históricos sobre el mismo tema; ambos a su vez son mínimos frente a otros corpus informativos. Por eso creemos que el estudio y la interpretación adecuada de estos procesos (productivos y de circulación), a través de las disciplinas históricas, tienen que establecer los mecanismos que nos permitan ordenar de manera integral, sobre la base de los datos empíricos y documentales con los que se cuenta hasta ahora, cada uno de los contextos de información, que al nivel de la arqueología se dan en: apropiación de recursos, procesos de transformación, producción, uso/función, re-uso, desecho y abandono.

Las habilidades y destrezas de la metalurgia, sobre todo en la confección de objetos y productos, queda demostrada tanto en las citas sobre el tema que brindan crónicas y documentos, así como en las innumerables colecciones, acervos de museos y hallazgos de excavaciones arqueológicas, sin embargo por ahora poco se sabe, y hablamos aquí específicamente en la zona del Perú, de las formas y modos de trabajo para la extracción de los minerales y las técnicas de refinamiento, fundición y labrado de metales y objetos. La mayor parte de estas formas y modos de trabajo se han inferido de los objetos recuperados.

En la búsqueda de estos conocimientos, la Arqueología no solo se basa en los datos recuperados en el campo, sino que, en algunos casos, ha pasado a terrenos experimentales (Shimada 1987) con relativo éxito y utilizando los elementos mas cercanos al contexto histórico que se estudiaba.

Ingresando al tema de las sociedades tardías del valle de Chincha ${ }^{1}$, nuestro trabajo se centra básicamente en la información recuperada en el sitio de Tambo de Mora (PV57-2), el cual posee ocupaciones Chincha, Chincha/ Inka y Colonial temprana (ca. 1200 d.C. hasta aprox. 1560 d.C.). Mucho se ha debatido sobre la sociedad Chincha y sus relaciones con la Inka tras la conquista de este valle por los cusqueños. A nivel etno-histórico, dos documentos "Aviso..." y "Relación...", han abierto las discusiones sobre la caracterización de la sociedad Chincha. Ambos documentos nos plantean una sociedad altamente compleja y jerarquizada dividida en tres grandes ámbitos económicos: la agricultura, la pesca y el intercambio, este último a larga distancia. Se habla también de productores especializados en la confección de ojotas, mates pirograbados, cerámica fina, textiles, entre otros. Destaca la mención de plateros u orfebres por su importancia en el ámbito tecnológico y económico para el intercambio. Una de las finalidades centrales de nuestro Programa de investigaciones, es el conocimiento integral de los modos de trabajo y de vida de la sociedad Chincha, por lo cual este dato se manifestaba de crucial importancia.

Las investigaciones arqueológicas por el momento, nos permiten afirmar la real existencia de Chincha como una sociedad altamente compleja. Los estudios en sitios de carácter habitacional de tipo popular y/o administrativo (caso Pampa de la Pelota), así como de ambientes pa-

1 Ubicado en la costa central andina, en el norte del departamento de Ica, Perú. 
laciegos y/o productivos (caso Tambo de Mora), confirman tal aseveración. A pesar de los avances obtenidos hasta el momento, los investigadores del tema, conspicuos o eventuales, siguen reduciendo el problema a dos aspectos que en el transcurso de las investigaciones, han devenido en cruciales, por lo menos para algunos: el mullu (Spondylus) como materia prima, bien o producto imprescindible para la existencia de la red de intercambio sobre todo con el norte, y en segundo lugar el o los otros productos por los cuales se intercambiaría.

Sin una visión global de la problemática se ha querido ver que los elementos primordiales del intercambio eran dos: el mullu y otro del mismo rango. Como contrapeso del mullu, se ha postulado innumerable variedad de productos incluyendo entre ellos el guano (Curátola 1997). Rostworowski sin embargo, basándose en lo que el propio documento "Aviso..." dice, plantea la intervención de variados productos en la red de intercambio, aunque también otorga un papel importante al mullu. Nosotros creemos que esta red de intercambio debió basarse en una multiplicidad de productos y materias primas que permitieran, no solo el interés de las sociedades en los extremos, y en los puntos intermedios, de esta red de intercambio, sino también los beneficios y privilegios para la sociedad Chincha, porque solo esto último es lo que permitiría su funcionamiento.

Para el tema específico de este alcance abordaremos el caso de los "plateros" y sus productos, para lo cual contamos con un corpus de trabajo preliminar. Revisando algunos datos previos para el valle encontramos que Uhle (1924) presenta, además de los datos referidos a cerámica, textiles y otros objetos (incluidos los metálicos), información gráfica y descriptiva de "fieles" de balanza o romanas ${ }^{2}$ provenientes de su excavación en el Cementerio B de La Cumbe, los cuales creemos que sirvieron en el inter-

2 Samano Xerez menciona que estas romanas servían para el intercambio de mullu. Nosotros pensamos que debió servir para diferentes cosas de gran valor, por ejemplo, como menciona Rostworowski "daban al oro un valor en relación a la plata diez veces el peso de la plata". cambio de metales preciosos, principalmente oro. Sandweiss (1992: 70-73), realiza una descripción de los objetos metálicos hallados en sus excavaciones del Sector I del sitio de Lo Demás, encontrando principalmente objetos de cobre, dentro de estos se menciona un fragmento de tupu de cobre. Un anzuelo de cobre, un pequeño cincel para el trabajo de metales y un fragmento de pinza decorada. Craig Morris, quien excavó en La Centinela, centro urbano capital de la sociedad Chincha, ha reportado la presencia de algunos objetos elaborados en metal.

Esta breve reseña de recurrentes presencias de metal no se diferenciaría mucho de cualquier otra sociedad de la misma época en otras regiones de la costa peruana. Sin embargo, la ubicación de una importante zona de hornos para el trabajo de orfebrería en el centro urbano de Tambo de Mora sí nos habla de una evidente especialización de "plateros" y del ingreso de nuevos productos y materias primas en la discusión e investigación de la red de intercambio a larga distancia de la sociedad Chincha.

\section{LAS EVIDENCIAS ARQUEOLOGICAS}

"... y tan bueno con todos los aderezos y herramientas que tienen. Y quando labran no hazen más de un hornillo de barro donde ponen el carbón, y con unos cañutos soplan en lugar de fuelles. ... por lo cual paresce ayer grandes plateros en este reyno." (Cieza de León, Crónica del Perú, 1553).

El Programa de Investigaciones Arqueológicas Chincha viene realizando estudios en el sitio de Tambo de Mora desde el año 1995. El enfoque de estos trabajos ha girado en torno a la recuperación de la información de los aspectos productivos, de organización y de ocupación del valle, a la vez que nos permita evaluar el potencial arqueológico del sitio respecto a la sociedad pre-colonial de los Chincha.

En el sitio de Tambo de Mora hemos realizado hasta el momento cinco temporadas de campo, interviniendo los sectores NW - Sub Sector Chacra, el sector SE - Sub Sector Recintos y el sector SW - Sub Sector "Hornos". En líneas 
generales, los sucesivos hallazgos y elementos identificados del sitio, reflejan una presencia importante de restos de actividades vinculadas a especialistas y, por lo tanto, de áreas dedicadas al almacenaje, preparación y consumo de alimentos en grandes cantidades (Sector NW Chacra W) y de trabajo de metales (Sector SW - Hornos).

\section{Sector SW - Sub Sector "Hornos"}

El Sector SW se caracteriza por presentar algunos pocos edificios de mediana altura, constituidos por plataformas y recintos de tapial y algunas estructuras bajas (SAN: 1942), entre estos edificios se identifican grandes espacios cercados al parecer por muros bajos. Este sector ha sido considerablemente afectado por la maquinaria pesada que ha cortado de NW a SE el área, exponiendo niveles de ocupación que formalizados dentro de recintos y cuartos cerrados y delimitados por muros de tapial. Al cortarse estos contextos, se dejaron al descubierto lentes de ceniza, deposiciones de consumo de peces, vegetales y moluscos, además de algunas estructuras de muros bajos y zonas de alta combustión focalizadas.

El Sub-Sector "Hornos", definido así por los hallazgos que ahora presentamos, se ubica al Este del sector, y se asocia a un edificio piramidal muy afectado desde 1995 (Fig.1) ${ }^{3}$. En esta zona se ubicó la Unidad 404 (trinchera prospectiva de 1 X 4 metros), que se orienta de $\mathrm{E}$ a W y está pegada al perfil expuesto.

La Unidad 404 buscaba registrar la gran concentración de lentes y capas que se quedaron evidenciados en un perfil producido por el corte de un sendero actual que, en este sector, pasa paralelo al gran perfil expuesto. La zona excavada tuvo, en sus primeros niveles, un área de menos de un metro cuadrado, lo que facilitó el trabajo de excavación y permitió evaluar detenidamente cada uno de los sedimentos identificados en la excavación. Hemos llegado a definir 26 eventos culturales (entre rellenos, fogones,

3 Los dibujos y fotografías pertenecen a los autores y han sido digitalizadas por Cecilia Núñez. lentes, hallazgos y otros), los cuales fueron registrados independientemente en 19 niveles de excavación que corresponden a las diferentes capas que hemos encontrado, denominadas de la letra "A" a la "Q". (fig.2).

Estos eventos han sido agrupados, luego de su evaluación, en 4 momentos culturales que, ordenados del más antiguo al más reciente, son:

1. Un primer momento asociado a época "Chincha", conformado por los niveles del 15M al 19Q (ambos incluidos). Se trata del interior del recinto SW, del montículo SW, compuesto por pisos y sucesivos rellenos hasta formar una especie de plataforma.

2. Un segundo momento de "Hornos" (Elemento 2), conformado por los niveles del $13 \mathrm{~K}$ y $14 \mathrm{~L}$, asociados con seguridad a época Chincha, aunque sin descartar que su uso se prolongue para los primeros momentos de la época Chincha/Inka.

3. Un tercer momento de "Basural", conformado por los niveles de 2B a 12J (ambos incluidos) y asociado a época Chincha/Inka. En el nivel 7E se identificó el Elemento 1, constituido por un hoyo con restos de basura y abundante material orgánico.

4. Un cuarto y último momento de Abandono/ Derrumbe, conformado por el primer nivel de la capa A.

\section{Elemento 2}

El segundo momento asociado a los "Hornos", lo constituyen capas de relleno, con material diverso a manera de lentes. En el nivel $13 \mathrm{~K}$ apareció el mencionado "Horno" identificado como el Elemento 2 (contexto 0456). Este horno presentaba revestimiento de barro, cocido por el uso, manteniendo en algunos sectores las improntas de los dedos del artesano. Debajo de este elemento, en el nivel 14L, se identificaron varios restos de hornos (hasta cuatro), similares al Elemento 2, pero distribuidos en diversos lugares y niveles, según el continuo uso de la zona. Estos hornos son de un nivel inferior y estaban muy alterados como consecuencia de 


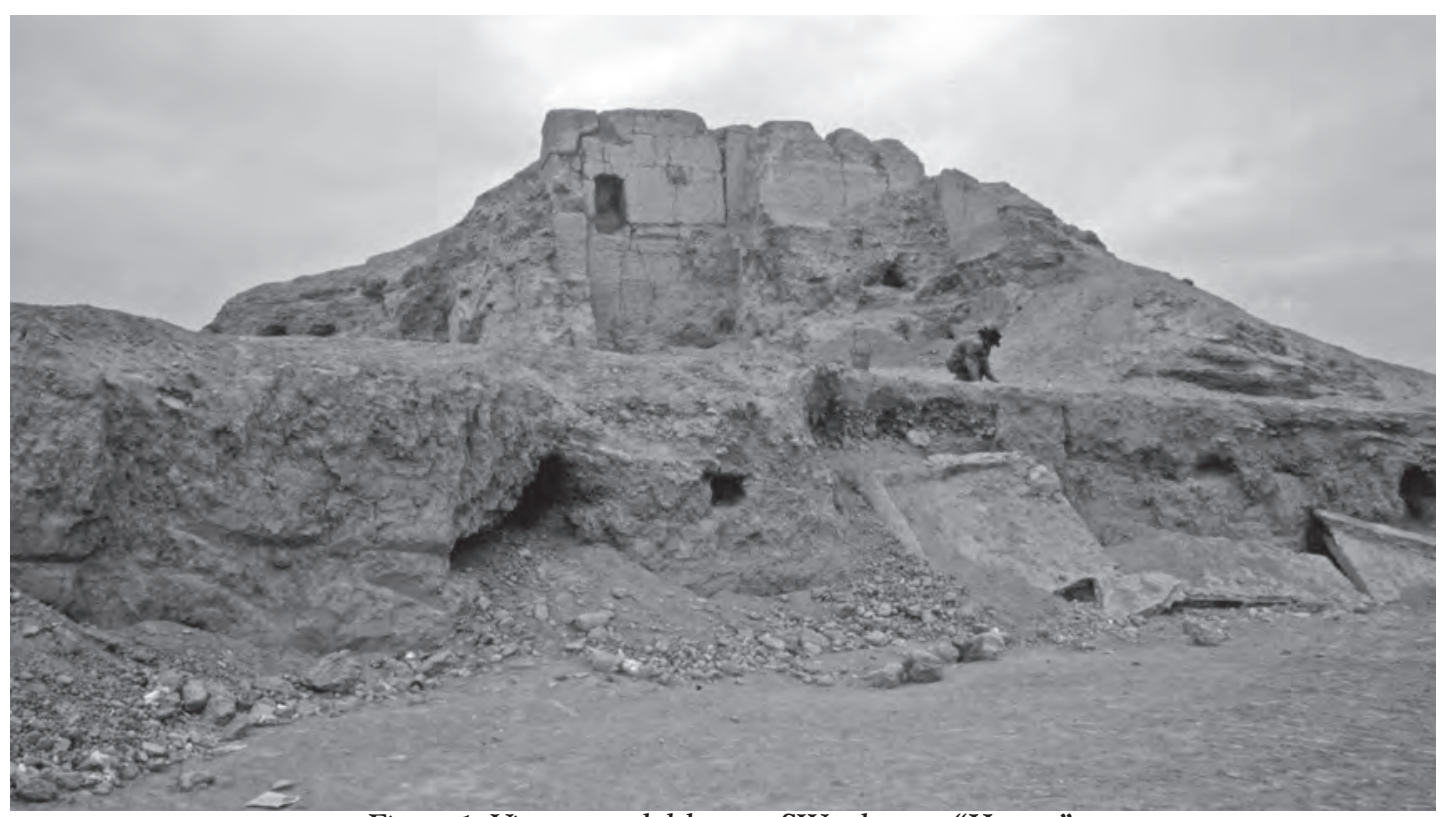

Figura 1. Vista general del sector SW-subsector "Hornos".

Como se puede apreciar, el sitio fue gravemente dañado por maquinaria pesada.
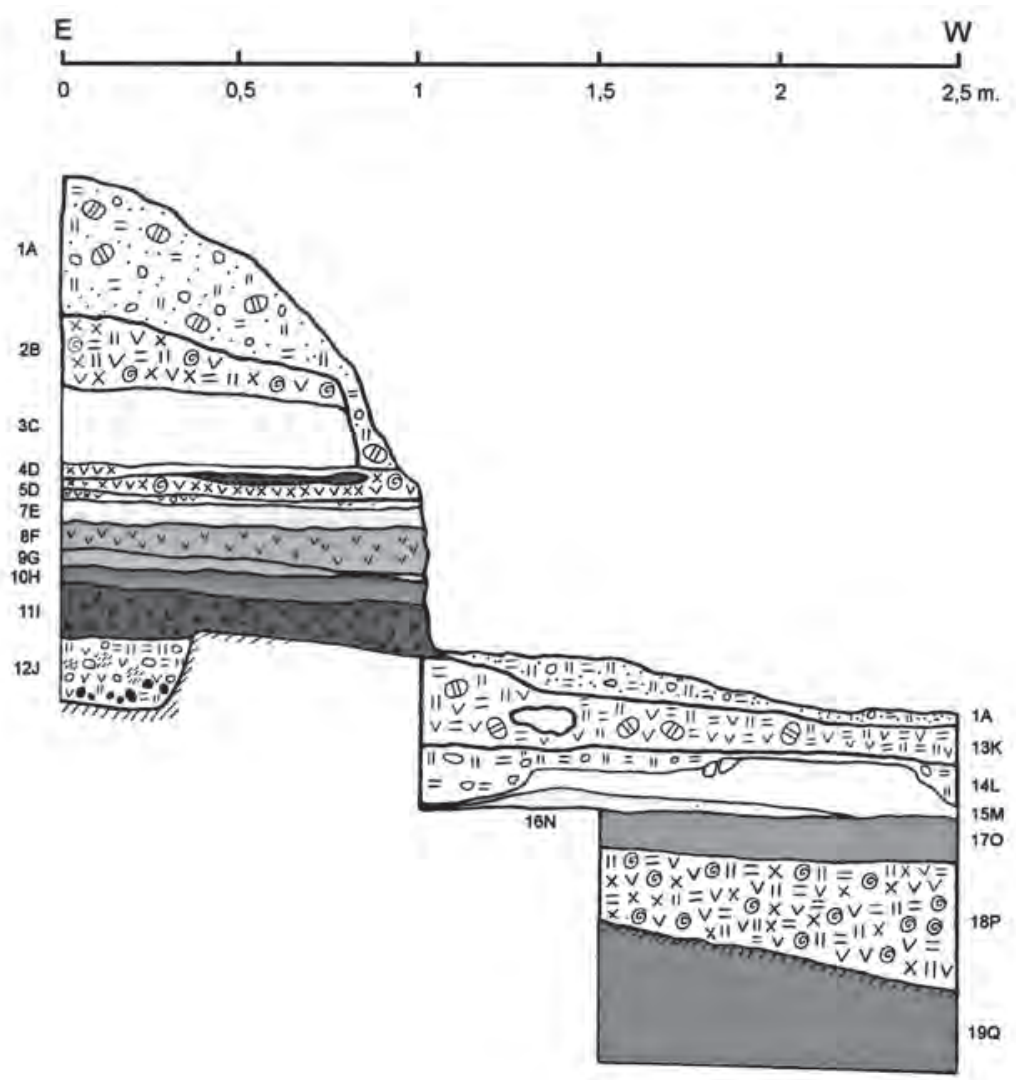

Figura 2. Corte/perfil esquemático del lado sur de la Unidad 404. Los restos de hornos se ubicaron, uno rellanado por 12J, y los otros en 13K y 14L; además, un depósito de carbón entre $17 \mathrm{O}$ y $18 \mathrm{P}$. 
la presencia del relleno $13 \mathrm{~K}$ y el sendero mencionado anteriormente. El horno presenta una forma ovalada, en su mayor parte (no se excavó totalmente), tiene un ancho promedio de 0.65 $\mathrm{m}$ y una extensión proyectada a $1.20 \mathrm{~m}$ aproximadamente. El extremo sur se encontró en mal estado de conservación.

\section{El Análisis de los materiales}

Durante las excavaciones de 1998, se recuperaron en la Unidad $402 \mathrm{CR}+$, en el nivel 2 de la capa $\mathrm{A}$, perteneciente a un contexto de compactación de escombros, un conjunto de fragmentos de tierra cocida los cuales son parte de moldes para metal y un fragmento de una probable "boquilla" de tubo para soplar al interior de los hornos (Fig. 3). Hay que señalar que estos contextos de procedencia se ubican a dos metros al N, y en el mismo nivel de ocupación que los hornos anteriormente descritos.

El primer detalle que llamó la atención de estos fragmentos, al margen de las figuras o líneas impresas, fue la presencia de zonas fuertemente termo-alteradas en el interior de los moldes y en el contorno del borde de la boquilla. Pasaremos a describir brevemente estos elementos:

TM 0428: En este contexto se halló un fragmento de molde, en cuya cara interna se observa un elemento figurativo. También se halló un fragmento de probable boquilla de tubo de ventilación. Estos tubos de ventilación servían

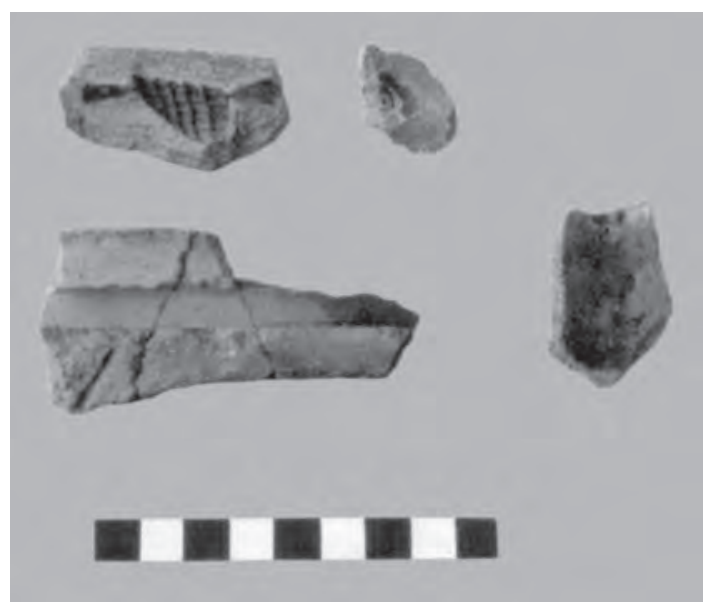

para insuflar aire en el horno soplando por un extremo (Benzoni, en Nordenskiöld 1921: 10).

TM 0429: 5 fragmentos de molde correspondientes a dos o tres moldes para metal. Uno de los moldes (incompleto) está conformado por tres fragmentos y presenta en el lado interno una acanaladura central y otra pequeña que comunica el centro con el exterior. Otros dos fragmentos de molde, probablemente del mismo, presentan elementos de carácter figurativo (ave o pez).

Una muestra de estos moldes fue sometida a análisis de microscopía electrónica obteniéndose los siguientes resultados:

MUESTRA 0M-00550: Corresponde a un fragmento de molde con código de procedencia 429 (Tambo de Mora, Sector SW. Unidad 402 nivel/capa 2/A, contexto de escombros/derrumbe). El análisis de esta muestra sirvió para la identificación de los metales que se utilizaron con el molde. La muestra presentó los siguientes componentes:

- Matriz: Arcillas con impurezas de $\mathrm{Cu}, \mathrm{Pb}$, Ag y Fe (80\%)

- Inclusiones: Sílice (8\%), plagioclasas (5\%), feldespato potásico $(5 \%)$, plata-cobre $(<$ $1 \%)$, cloruro de plata $(<1 \%)$, oro $(<<$ $1 \%)$, plomo-antimonio $(<<1 \%)$, plomovanadio-cobre-hierro $(<<1 \%)$.

Como se puede observar, la muestra está constituida básicamente por arcillas, que son el cuerpo mismo del molde, que han presentado impurezas de plomo, cobre, fierro y también de plata. Estas arcillas suelen contener inclusiones de sílice-plagioclasas de tamaños menores a cien (100) micrones, de sílices de tamaños menores a ochenta (80) micrones, y de feldespatos (feldespatos potásicos y plagioclasas) de tamaños menores a veinte (20) micrones. Además de estas inclusiones, se han observado inclusiones metálicas de plata y de aleación plata/cobre de tamaños menores a quince (15) micrones. De mane-

Figura 3. Vista del conjunto de moldes (procedencia 429) a la izquierda, y fragmento de probable "boquilla" para ventilación a la derecha (procedencia 428). 
ra menos frecuente se han observado presencias de cloruro de plata de tamaño menor a quince (15) micrones, también de oro en tamaño menor a ocho (8), así como de plomo-antimonio de tamaño menor a veinte (20) micrones, y de plomo-vanadio-cobre-hierro de tamaño menor a cinco (5) micrones. El plomo-cobre, además de inclusiones, suele encontrarse en forma de venillas cortando las arcillas.

MUESTRA 0M.00550 A: Corresponde a un fragmento del cuerpo del molde anteriormente descrito, es decir, a la parte interna de la masa de arcilla. Este análisis se efectuó a fin de establecer comparaciones con las pastas cerámicas tardías de Chincha, que serán sometidas a análisis similares en futuras temporadas de trabajos. Estas comparaciones nos servirán para determinar la relación que puedan tener estos moldes con la producción alfarera de vasijas.

La muestra está constituida por fenos de cuarzo, menores a cien (100) micrones, y playas de epídota de tamaños menores a trescientos (300) micrones. Menos frecuentes son los cristales listonados de cloritas, menores a cien (100) micrones, así como los cristales anhedrales de feldespatos y piroxenos menores a cincuenta (50) micrones, todos ellos envueltos por una matriz de arcilla y sílice. Esta muestra presenta además pequeñas diseminaciones de ilmenita. Como se observa el interior del molde no presenta inclusiones de plata y/o plata-cobre.

Otros materiales analizados: en el marco del Programa de investigaciones, y procedentes de otros sectores del sitio, pero de las mismas épocas, hemos identificado otras evidencias sobre metales. Si bien las identificaciones preliminares se hicieron desde el punto de vista macroscópico, el potencial de los restos identificados, recuperados durante todas las campañas, permitirá reforzar aspectos inherentes a la actividad especializada en trabajos orfebres. El material procede de 42 contextos que presentaron evidencias de metales y asociados. Lo significativo del muestrario es la importante presencia de plata (en muchos casos en aleación con cobre).

Del cien por cien del muestrario total de metales asociados, un $42.86 \%$ corresponde a restos de escorias (que serán analizadas en otra oportunidad). Luego, un 29.58\% corresponden a restos metálicos, entre los que tenemos identificados al cobre $(23.82 \%)$ y la plata $(4.76 \%)$, todos estos manifestados como restos de mineral en bruto. El 28.56\% restante del muestrario corresponde a instrumentos metálicos, principalmente fabricados en cobre con un $11.90 \%$ y que consiste en 3 fragmentos de pinzas, 1 fragmento de lámina y 1 anzuelo que al parecer es bastante tardío. Luego vienen los instrumentos de plata con un $9.52 \%$ de presencia, representado por dos fragmentos de láminas, una cuenta romboidal entera y en buen estado de conservación y un fragmento de aguja, y finalmente, compartiendo el mismo porcentaje de presencia $(2.38 \%)$, tenemos un fragmento de lámina de oro/cobre (tumbaga), un disco de hematina y restos de material ferroso, que al parecer es intrusivo.

Durante las investigaciones de otros materiales recuperados en el sitio Tambo de Mora, se ha podido detectar, entre otras cosas, una pequeña lámina (0132) en forma de media luna, de borde irregular, compuesta por una cara de plata y la otra de oro. La técnica de confección es la de laminado y recorte, y corresponde a plata socapada de oro. Así mismo, se han ubicado una aguja (0135) de plata trefilada, cinco pequeñas láminas de plata, y una pequeña lámina discoidal con punto embutido cerca del centro, confeccionada en tumbaga. También se ha podido observar fragmentos de piezas de cobre confeccionadas en vaciado.

\section{Instrumentos líticos asociados}

Todos los materiales arqueológicos líticos de las tres temporadas de excavación realizadas en el sitio de Tambo de Mora (PV57-2), conforman un universo de 247 especimenes y fueron sometidos a un estudio macroscópico detallado en lo que se refiere a su funcionalidad, elaboración y materia prima, quedando pendiente la identificación completa de los materiales y los análisis microscópicos necesarios. De todos los materiales recuperados, los que interesan al presente estudio son: 
Instrumentos Abrasivos: Esta clase de instrumentos se usaron para desbastar, alisar y pulimentar materiales tales como la madera y las conchas, aunque es posible que también se usaran para trabajos relativamente finos en la metalurgia. Dentro del universo de la muestra, esta clase representa casi el $29 \%$ y se divide en los siguientes tipos:

- Alisador: Representa el 15.74\% de la muestra. Elaborado de arenisca $(1.21 \%)$, basalto $(0.41 \%)$, conglomerado $(0.41 \%)$, cuarcita (2.38\%), no identificados $(2.02 \%)$ y piedra pómez (9.31\%).

- Pulidor: Representa el $12.14 \%$ de la muestra. El tipo presenta diversas formas, pero el tamaño siempre es el adecuado para sujetarlo con una mano y tener la superficie activa, plana y lisa. Probablemente se utilizó para dar acabado final a algunas cerámicas $\mathrm{u}$ otros objetos como metales y maderas. La materia prima usada a sido cuarcita (5.26\%), basalto $(2.03 \%)$ y no identificado $(5.26 \%)$.

Instrumentos percutores: Estos instrumentos se caracterizan básicamente porque su extremo distal puede ser romo, plano o ahusado; el material puede ser blando o duro, dependiendo de la labor que se vaya a ejecutar, pero básicamente es el de debastar y dar un retoque más o menos grueso. En este caso también se han incluido en esta clase diversos tipos de instrumento que por su forma de uso siempre utilizan la fuerza cinética:

- Yunque: Representa el 0.80\% de la muestra. Este tipo de elemento pasivo está preparado en cuarcita. La forma observada es de una piedra de superficie de trabajo plano (presenta desgaste), mas o menos amplia. Uno de los especimenes es relativamente pequeño, como el tas de un orfebre.

- Percutor: Representa el $0.80 \%$ de la muestra. Formalmente es casi cilíndrico con el extremo distal ligeramente romo o plano y el proximal para ser sujetado por los dedos. Para el presente caso, son pequeños, como para retoque o laminado. La materia prima empleada es cuarcita.

- Mesa de Trabajo: Representa el 2.02\% de la muestra. Es un instrumento pasivo de superficie plana y medianamente amplia. En ella se notan las trazas de cortes y percusiones y alguna que otra huella de quemaduras o restos de resinas. Los especimenes fueron hechos de cuarcita.

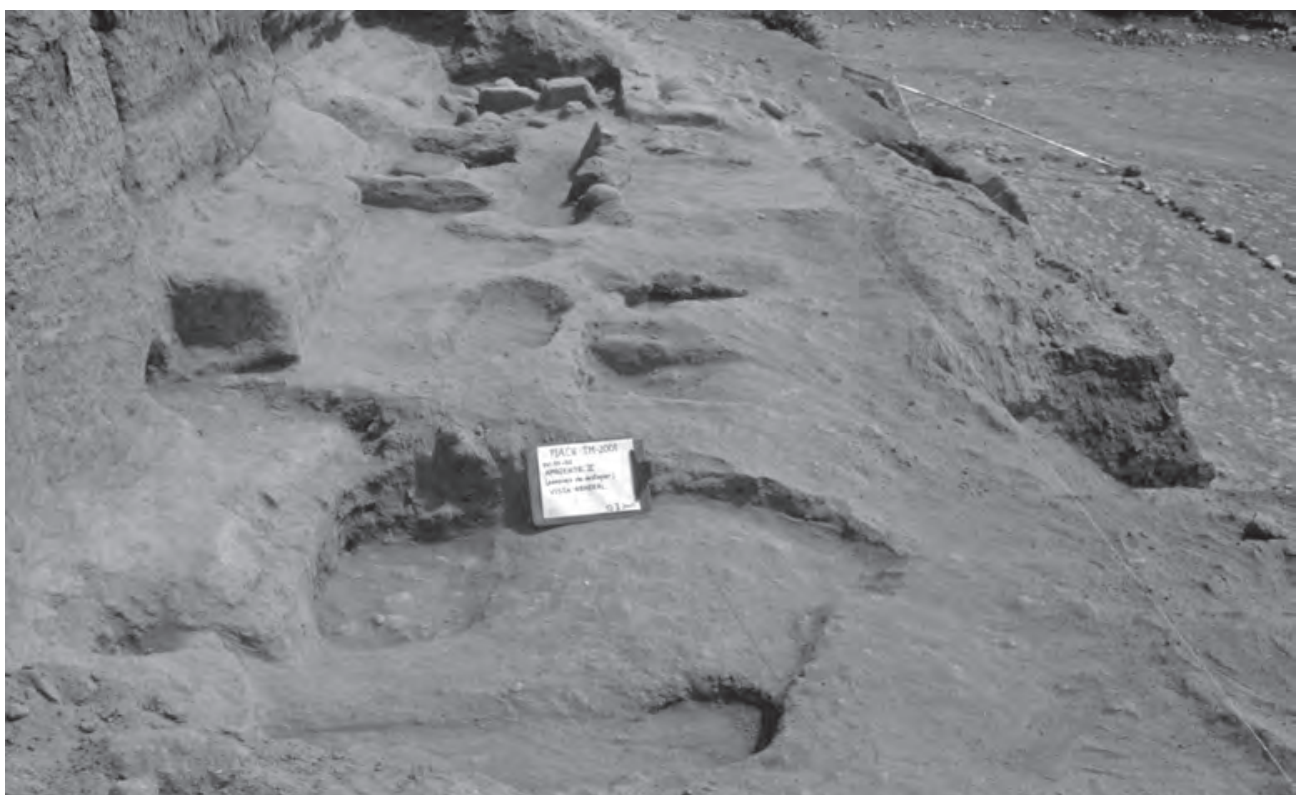

Figura 4. Tambo de Mora, subsector "Hornos", ambiente 2. Vista general de la última ocupación del taller de orfebrería 

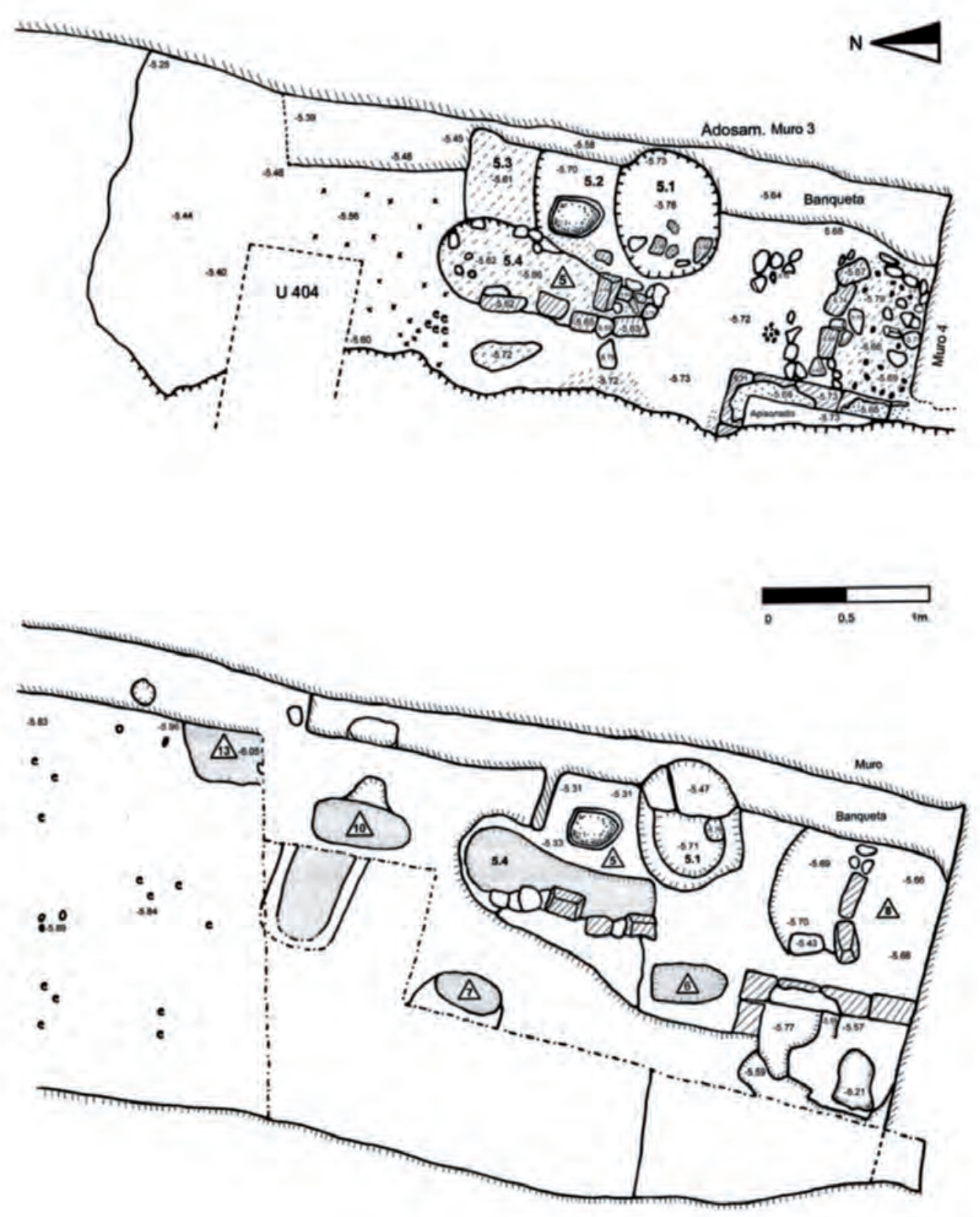

Figura 5. Tambo de Mora, subsector "Hornos", ambiente 2. Vistas sucesivas del proceso de excavación en las que se notan las diferentes áreas presentes en el taller de orfebrería. El Elemento 5 integra el área de trabajo de orfebrería por calentamiento: horno de calentamiento (5.4); mesa de trabajo (5.2); hoyo de arena (5.1). En los alrededores se pueden observar los hornos de refinamiento $(6,7,10,13$ entre otros), así como el depósito de carbón. 


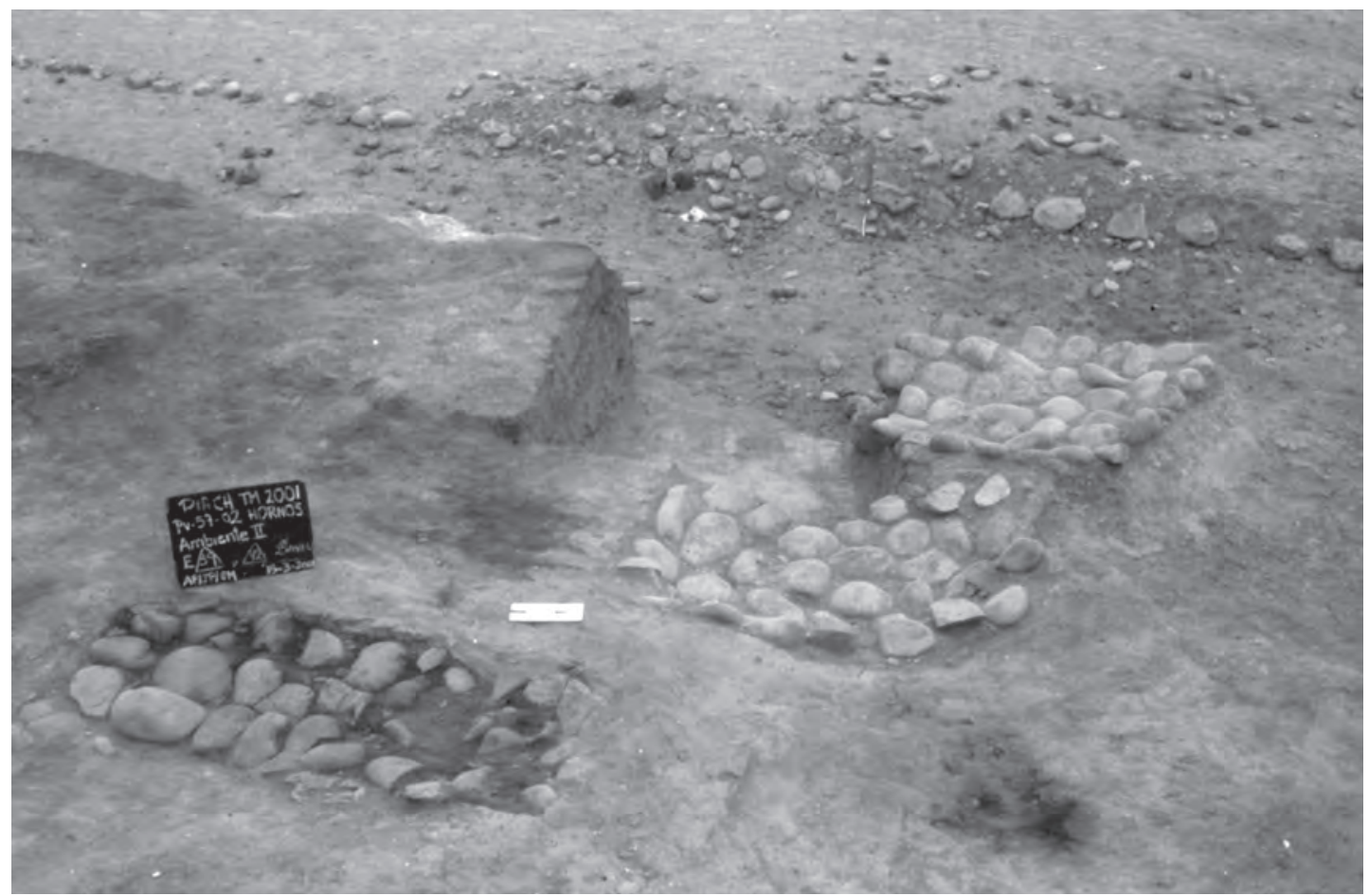

Figura 6. Tambo de Mora, subsector "Hornos", ambiente 2. Depósitos de carbón conformados por una base y pequeño murete de cantos rodados. Los de la fotografía corresponden a los primeros niveles de ocupación de los talleres.

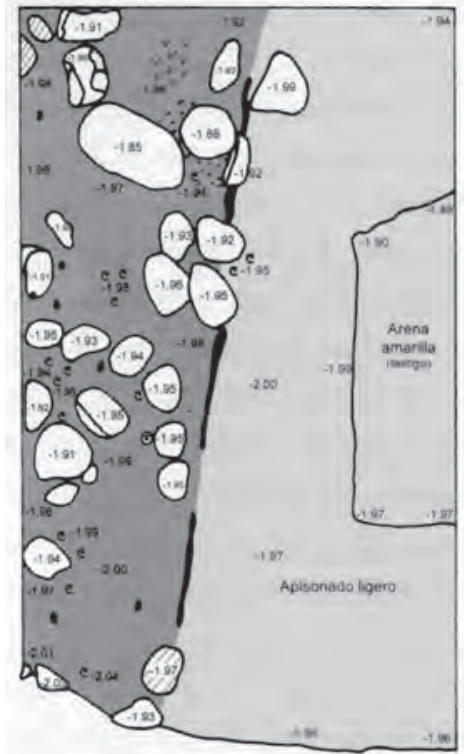

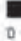

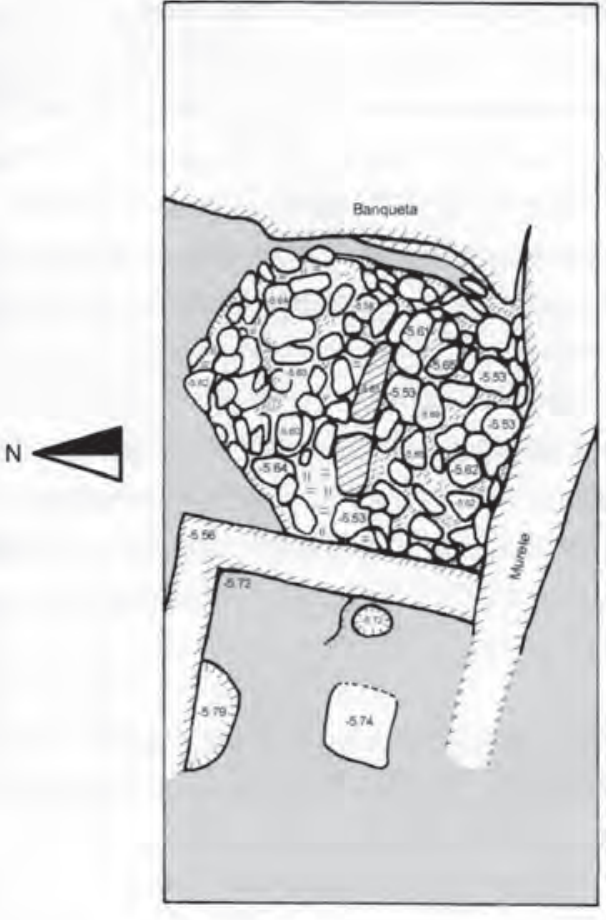

Figura 7. Tambo de Mora, subsector "Hornos", ambiente 2. El gráfico de la izquierda corresponde a los restos de depósitos de carbón de la Unidad 404, y pertenece a los primeros niveles de la ocupación de los talleres. A la derecha, la Unidad 415, elemento 8. Depósito de carbón subdividido en dos partes. Pertenece a la última ocupación de los talleres. 
La ampliación de las excavaciones durante las temporadas 1999 y 2000 nos han permitido, en función de la inicial Unidad 404, recuperar una serie de talleres metalúrgicos, algunos de los cuales se hallaban bastante bien conservados, sobre todo los de la última ocupación (Fig.4). Una meticulosa excavación y un exhaustivo registro de la misma nos vienen permitiendo establecer la secuencia de sucesivos remodelamientos, re-usos y redistribuciones del área de taller (Fig.5). Entre los diversos elementos que conforman los talleres, los que mas destacan por su nivel de conservación y por su abundancia son los depósitos de carbón (Figs. 6 y 7). Como se puede observar en las últimas temporadas, las excavaciones han resultado fructíferas y han permitido la recuperación de gran cantidad de información que permite confirmar y afinar la hipótesis de una especialización de alto nivel en platería en el Señorío de Chincha.

\section{LAS EXCAVACIONES Y los “plateros" De Chincha}

A manera de puntos de discusión, presentaremos algunos de los resultados y discusiones que en torno a las investigaciones se han venido dando:

1.- Como se viene demostrando por las evidencias y por la elaboración de los datos, la especialización en platería es indiscutible. Sin embargo, no hay que perder de vista que para la sociedad Chincha tardía existen evidencias de otras múltiples y también complejas actividades económicas, que van desde lo agropecuario, pasando por la pesca y la extracción del guano hasta la manufactura y producción de una multiplicidad de bienes (cerámica, ojotas, mates, útiles de madera, etc.).

2.- considerando que una parte importante de la sociedad Chincha estaba dedicada al intercambio, tal como señalan los documentos etno-históricos al hablar de mercaderes, se debe entender que este constituía una fuerte de recursos y beneficios que permitían la propia sustentación del intercambio. Otra consideración a tener en cuenta es que muy probablemente casi el tercio de la población de Chincha dedicada al intercambio no lo hacía de manera directa, existirían subdivisiones al interior como los "mercaderes" propiamente dichos (representantes de curacas y señores), los navegantes, los porteadores y caravaneros y los artesanos y productores de bienes transformados (el aporte de productos de la pesca y las actividades agropecuarias es también de relevancia). En función de esto suponemos que las evidencias de un taller especializado, al interior de una sociedad con capacidad de obtener esos bienes por el intercambio, indicaría que su producción era rentable y que estaría, por lo menos en parte, dedicada al "mercadeo" que permitía el acceso a otros recursos, o a situaciones de prestigio con las consiguientes prerrogativas sociales en los ámbitos por donde se extendiera la red de intercambios.

3.- Las excavaciones en esta zona del sitio, asó como en otras zonas del mismo y de otros sitios, nos vienen demostrando drásticas modificaciones al interior de los asentamientos. Estas modificaciones son notables en el paso de la época Chincha a la Chincha/ Inka. La zona de estudio del presente trabajo es un ejemplo de lo dicho, pues sobre un recinto de amplias dimensiones dedicado al trabajo en hornos para metales, se suponen elementos arquitectónicos más restringidos y una zona de basural. El cambio de uso de la zona así como los materiales asociados,, nos permiten pensar que la especialización de los "plateros" existe desde la época Chincha, con una potencialización de la actividad en época Chincha/Inka con un cambio de uso en plena época de control Inka. La envergadura del traslado de los talleres es muy difícil de determinar por ahora, existiendo dos opciones posibles: en el mismo valle hacia un sitio de control directo de la administración Inka, o, como algunos documentos indican, hacia la región del Cuzco, 
donde fueron trasladados gran cantidad de artesanos y orfebres especializados.

4.- La zona de hornos presenta en la actualidad un potencial de tamaño cercano a los 100 metros cuadrados, sin embargo, hay que considerar la zona pedida por la destrucción con maquinaria. A esto hay que sumar que en la pequeña área de exposición de la excavación se han ubicado hasta nueve hornos. Esto habla claramente de la intensidad de los trabajos en estos talleres. En cuanto a esto, hay que señalar que existen tres grandes momentos de superposición de talleres totalmente remodelados. Sin embargo, al interior de los mismos existen evidencias de uso, abandono, remodelación y redistribución de hornos que también indican la intensidad del trabajo en los en los talleres.

5.- Las características morfo-funcionales de los referido hornos nos indican que estos elementos son del tipo de horno para calentamiento y también para refinamiento o "purificación" (mas no de "recuperación"). El estudio posterior de los fundentes asociados a las escorias nos permitirá verificar este tipo de trabajo.

6.- El análisis formal de los hornos, así como el de las evidencias asociadas y el análisis de microscopía electrónica, demuestran un corpus coherente para el trabajo de metales por orfebres o "plateros". El sistema de trabajo en los talleres es todavía fácilmente observable en muchas comunidades andinas, tanto así que se han efectuado estudios recientes en plateros aymaras de Bolivia que trabajan en un sistema muy parecido pero con instrumentos "modernos" (Morssink 2000).

7.- Las técnicas empleadas por los orfebres de Chincha para la confección de objetos, según las evidencias arqueológicas encontradas, son las de moldeado y laminado por martilleo. El uso de aleaciones era bastante común (p.e. tumbaga), así como las diferentes maneras de enchapes (oro/plata).
8.- La presencia de combustible en el fondo de algunos hornos nos indica el uso de carbón hecho a base de especies nativas del bosque ribereño y bosque xerófilo, principalmente guarango, aunque en algunos momentos, o fases de trabajo, se pudo dar el uso de leña de igual procedencia.

9.- El control de temperatura en los hornos debió ejecutarse siempre con tubos insufladores con boquillas ya que los ambientes de los talleres son semiabiertos y los hornos no guardan ninguna disposición en particular con los vientos (los hornos tienen diferentes alineaciones). Esto confirma aún más que se tratan de hornos de taller de orfebrería, a diferencia de los hornos de recuperación, conocidos como guairas, y que siempre se colocaron en partes altas y orientadas hacia los vientos, teniendo como combustible la leña y estiércol (Capoche 1585).

10.-Una pregunta aún sin respuesta satisfactoria es la proveniencia de los minerales y metales. En la actualidad no se conocen minas de plata o de oro en el valle de Chincha, sin embargo es posible que, al igual que en otras zonas de los Andes (Angiorama, Ms. 1999), los yacimientos de oro y plata nativa se encuentren en las zonas altas y de puna, aunque en el caso del oro, tampoco se descartaría su extracción mediante "aventaderos" y "veneros" en valles medios y bajos. Lo que sí es seguro es que la primera molienda del mineral, así como el uso de hornos de recuperación, principalmente para el cobre, se realizaría cerca de los lugares de extracción, lejos de los lugares de manufactura. Se conocen asentamientos con presencia de cerámica Chincha y Chincha/Inka en las zonas altas del valle, y aunque el resto del patrón de asentamiento es totalmente diferente, la presencia en la zona, directa o indirecta, está asegurada. Esta presencia, entre otras cosas, garantizaría el acceso a los minerales y metales de las zonas altas de Chincha y Huancavelica. 


\section{BibLIOGRAFía}

Alcalde, Javier y Carlos Del Águila

1999 "Moldes para metal y análisis de microscopía electrónica de muestras procedentes de Tambo de Mora (PV57-2)". En Investigaciones Arqueológicas en el Valle de Chincha. Informe final 1998. Instituto Andino de Estudios Arqueológicos - INDEA y American Museum of Natural History. Lima.

Angiorama, C. I.

1999 "Metales y metalurgos en la quebrada de Humahuaca prehispánica (Jujuy, Argentina)". Presentado a Arqueología Espacial, Seminario de Arqueología Turolense, Teruel. Manuscrito.

Anónimo

1977 Aviso del modo que avia en Gobierno de los indios en tiempo del Inga y como se repartian las tierras y tributos. Biblioteca del Palacio Real de Madrid, Miscelánea de Ayala/Tomo XXII, folios 261. 273 v. En Etnía y Sociedad: 130-140. Instituto de Estudios Peruanos. Lima.

Capoche, L.

1585 "Relación general de la Villa Imperial de Potosî", pp. 108-124, en Textos y documentos de la América Hispánica (1492. 1898). Historia de España, Tomo XIII, Manuel Muñón de Lara (editor) (1988), pp. 161-164. Ed. Labor. Barcelona.

Cieza de Leon, $\mathrm{P}$.

1984 Crónica del Perú. Fondo Editorial de la Pontificia Universidad Católica del Perú. Lima.

Crespo, J. C.

1975 "La Relación de Chincha (1558)”. En Historia y Cultura 8: 91-108. Lima.

Curatola, M.

1997 "Guano: una hipótesis sobre el origen de la riqueza del señorío de Chincha". Arqueología, Antropología e Historia en los Andes. Homenaje a María Rostworowski. R. Varón y J. Flores Ed. Instituto de Estudios Peruanos. Lima.
Fujita, Fernando

1999 "Análisis de materiales líticos recuperados en el sitio de Tambo de Mora (PV57-2)". En Investigaciones Arqueológicas en el valle de Chincha. Informe final 1998: 119-134. Instituto Andino de Estudios Arqueológicos - INDEA y American Museum of Natural History. Lima.

González Luis R. y Ana M. Vargas

2000 "Tecnología metalúrgica y organización social en el noroeste argentino prehispánico. Estudio de un disco”. En Chungara 31/1: 5-28. Universidad de Tarapacá, Chile.

Instituto Andino de Estudios Arqueologicos (INDEA)

1999 Investigaciones Arqueológicas en el valle de Chincha 1998. Informe Final, Programa de Investigaciones Arqueológicas Chincha. Lima. Manuscrito.

Lumbreras, L. G.

2001 "Uhle y los asentamientos Chincha en el siglo XVI". En Revista del Museo Nacional XLIX: 13-87. Lima.

Morssink, Rob A.

2000 "La plata en las culturas andinas". En Chungara 31/1: 49-80. Universidad de Tarapacá, Chile.

Nordenskiöld, E.

1921 "The copper and bronze ages in South America". Comparative Ethnographical Studies, 4. Göteborg.

Relación

1920 Relación del origen e govierno que los ingas tuvieron, y del que habia antes que ellos señoreasen a los indios deste Reyno, y de qué tiempo, y de otras cosas que el gobierno conuenia declaradas por señores que sirvieron al Inga Yupanqui, y a Topa Inga Yupanqui y a Guana Capac y Guascar Inga (1558). En Col. De Lib. Referentes a la Historia del Perú. Segunda Serie. Lima. 
Retamozo, Enrique y Carlos Del Águila

1999 "Evaluación preliminar de metales y/o restos minerales del sitio Tambo de Mora (PV57-2)”. En Investigaciones Arqueológicas en el valle de Chincha. Informe final 1998: 136-138. Instituto Andino de Estudios Arqueológicos INDEA y Américan Museum of Natural History. Lima.

Rostworowski, M.

1977 Etnía y Sociedad, Costa Peruana Prehispánica. Instituto de Estudios Peruanos. Lima.

Sandweiss, D.

1992 "The Archaeology of Chincha Fishermen: Specialization and Status in Inka
Peru". En Bulletin of Carnegie Museum of Natural History 29: 70-73. Pittsburgh.

Shimada, I.

1987 "Aspectos Tecnológicos y Productivos de la Metalurgia Sicán, costa norte del Perú”. En Gaceta Arqueológica Andina 13: 15-21. Instituto Andino de Estudios Arqueológicos (INDEA). Lima.

Uhle, $\mathrm{M}$.

1924 "Explorations at Chincha. University of California Publications" in American Archaeology and Ethnology, 21 (1): 57-94. California. 